\title{
Smartphone-based Traffic Information System for Sustainable Cities
}

\author{
Vasileios Manolopoulos, Sha Tao, Ana Rusu, and Panos Papadimitratos \\ \{vama;stao;arusu;papadim@kth.se\} \\ KTH Royal Institute of Technology, Stockholm, Sweden
}

Smartphones have several advantages for data collection in traffic information systems (TISs). Smartphone-based TISs (Fig. 1 shows such a system) can have significantly lower cost and larger coverage compared to TISs based on roadside infrastructures or dedicated on-board units. The increasing smartphone penetration has also the potential of providing a huge traffic probe base.

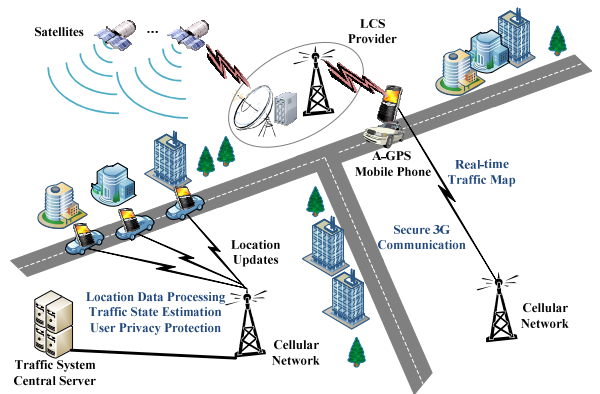

Fig. 1. Smartphone-based Traffic Information System

Towards a fully operational system of this type, a number of issues must be addressed [1], notably: (i) security and privacy, and (ii) processing of the collected location data to estimate the traffic state, especially for urban road networks. With several TIS applications and a few field tests, this demonstrator brings forth novel features. Based on our results [1][3], we implemented and integrated all necessary components to get a system that: (i) estimates traffic conditions solely based on data from smartphones, (ii) collects data securely to protect the TIS, (iii) does so in a privacy preserving manner; and our system achieves (ii) and (iii) by extending the existing 3G cellular authentication architecture.

Demonstrator Overview: Our demonstrator consists of three components: (i) the Android application running on drivers' smartphones, (ii) the traffic server, and (iii) the authentication server. The application sends location updates to the traffic server and it requests traffic estimates for the nearby area. The server gathers location data and sends the estimated traffic states to drivers.

User Functionality: When launching the application, a user can choose between different scenarios. In each scenario a pre-recorded trace of an emulated probe in the selected area is played. The smartphone's position is displayed on a map. Through the application, the user can set the time period for computing his/her location, the period for sending this location update to the server and the period for requesting traffic updates from the server. The received traffic states from the server are classified into three traffic condition levels: 1) green level (smooth traffic) if speed is more than $25 \mathrm{~km} / \mathrm{h}, 2$ ) orange level (medium traffic) if speed is between 14$25 \mathrm{~km} / \mathrm{h}$, and 3) red level (congested traffic) if speed is less than $14 \mathrm{~km} / \mathrm{h}$. As shown in Fig. 2, the driver is able to reroute his/her journey, i.e., choose the optimal path, based on the updated traffic conditions presented on the smartphone's display (as colored road segments).

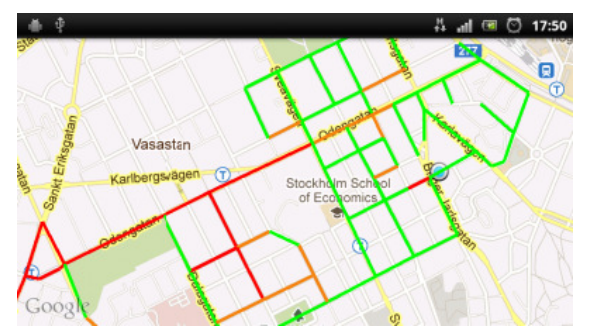

Fig. 2. Screenshot of the Android Application

Server Functionality: The traffic estimation integrated in this demo is based on a simulation testbed [2]. The emulated smartphone location updates are processed first and then allocated to nearest road links; the aggregate speed estimates on each road link are estimated. The probe traces displayed in the scenarios are recorded from a microscopic traffic simulation. All communication functionality, including the security and privacy mechanisms are implemented together with anonymous authentication running on the smartphones [3]. Mutual authentication and encryption are used and the traffic server verifies that the incoming message is from an authorized user; however the user remains anonymous within the group of legitimate users.

Demo Setup: The two servers will run locally in a laptop and the smartphones will communicate over $\mathrm{WiFi}$ for convenience and to ensure operation in demo conditions. Internet access will be needed.

\section{REFERENCES}

[1] V. Manolopoulos, S. Tao, S. Rodriguez, M. Ismail and A. Rusu, "MobiTraS: A Mobile Application for a Smart Traffic System," in the $8^{\text {th }}$ IEEE NEWCAS Conference, June 2010, Montreal, pp. 365-368.

[2] S. Tao, V. Manolopoulos, S. Rodriguez, and A. Rusu, "Real-time Urban Traffic State Estimation with A-GPS Mobile Phones as Probes," in SCIRP Journal of Transportation Technologies, in press.

[3] V. Manolopoulos, P. Papadimitratos, S. Tao, and A. Rusu, "Securing Smartphone Based ITS," in the $11^{\text {th }}$ IEEE ITST Conference, September 2011, St. Petersburg, pp. 201206. 